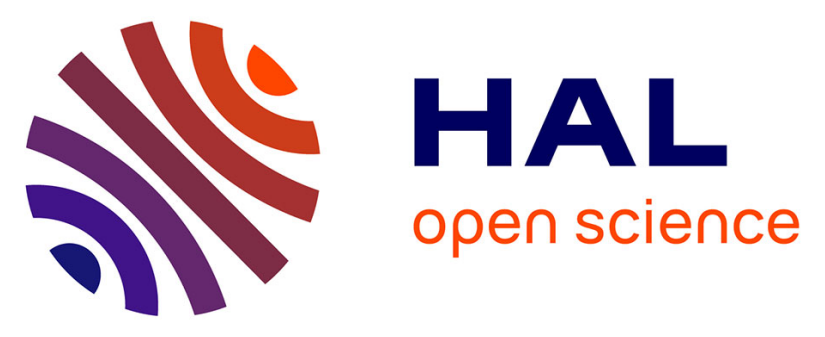

\title{
Unexpected categories at risk of S. aureus nasal carriage among hospital workers
}

Sandrine Boisset, Mitra Saadatian-Elahi, Caroline Landelle, Michèle Bes, Claude-Alexandre Gustave, Anne Tristan, Jean-Baptiste Fassier, Frédéric Laurent, Jacqueline Grando, François Vandenesch, et al.

\section{To cite this version:}

Sandrine Boisset, Mitra Saadatian-Elahi, Caroline Landelle, Michèle Bes, Claude-Alexandre Gustave, et al.. Unexpected categories at risk of S. aureus nasal carriage among hospital workers. International Journal of Hygiene and Environmental Health, 2019, 222 (8), pp.1093-1097. 10.1016/j.ijheh.2019.08.003 . hal-02352128

\section{HAL Id: hal-02352128 \\ https://hal.science/hal-02352128}

Submitted on 28 May 2021

HAL is a multi-disciplinary open access archive for the deposit and dissemination of scientific research documents, whether they are published or not. The documents may come from teaching and research institutions in France or abroad, or from public or private research centers.
L'archive ouverte pluridisciplinaire HAL, est destinée au dépôt et à la diffusion de documents scientifiques de niveau recherche, publiés ou non, émanant des établissements d'enseignement et de recherche français ou étrangers, des laboratoires publics ou privés. 
Unexpected categories at risk of $S$. aureus nasal carriage among hospital workers

Sandrine BOISSET ${ }^{(1,5)}$, Mitra SAADATIAN-ELAHI ${ }^{(2,3)}$, Caroline LANDELLE ${ }^{(4,5)}$, Michèle BES $^{(2,6)}$, Claude-Alexandre GUSTAVE ${ }^{(2,6)}$, Anne TRISTAN ${ }^{(2,6)}$, Jean-Baptiste FASSIER ${ }^{(7)}$, Frederic LAURENT ${ }^{(2,6)}$, Jacqueline GRANDO $^{(8)}$, François VANDENESCH $*(2,6)$, Coralie BOUCHIAT $*(2,6)$

* equal senior authorship

${ }^{(1)}$ Laboratoire de bactériologie, Centre Hospitalier Universitaire de Grenoble Alpes, France

${ }^{(2)}$ Centre National de Référence des Staphylocoques, Institut des Agents Infectieux, Hospices Civils de Lyon, Lyon, France

${ }^{(3)}$ Service d'Hygiène, Epidémiologie et Prévention, Hospices Civils de Lyon, F-69437 Lyon, France

${ }^{(4)}$ Service d'hygiène hospitalière, Centre Hospitalier Universitaire de Grenoble Alpes, France (5) Univ. Grenoble Alpes, CNRS, Grenoble INP, CHU Grenoble Alpes, TIMC-IMAG, F38000 Grenoble, France

(6) CIRI, Centre International de Recherche en Infectiologie, Université de Lyon; Inserm U1111; Ecole Normale Supérieure de Lyon; Université Lyon 1; CNRS, UMR5308, Lyon, France

(7) Univ Lyon, Université Claude Bernard Lyon1, Ifsttar, UMRESTTE, UMR T_9405, F69373, LYON ; Service de médecine et santé au travail, Hospices Civils de Lyon, France

${ }^{(8)}$ Unité d'Hygiène Hospitalière, Hospices Civils de Lyon, France 


\section{Corresponding author}

Dr Coralie Bouchiat

Laboratoire de bactériologie, Institut des agents Infectieux, Hospices Civils de Lyon, Lyon,

France

Centre National de Référence des Staphylocoques, Lyon, France

Faculté de medicine de Lyon Est, Université Lyon 1

Centre International de Recherche en Infectiologie, Unité INSERM U1111, CNRS

UMR5308, Université de Lyon, Ecole Normale Supérieure de Lyon

Phone: $+33(0) \cdot 4 \cdot 72 \cdot 07 \cdot 16.95$

Fax: +33(0).4.72.07.18.42

Mail: coralie.bouchiat@chu-lyon.fr 


\section{ABSTRACT}

Objectives. Thirty percent of the general population are Staphylococcus aureus nasal carriers. It has been shown that this increases with repeated contact with patients, but it is not known whether all categories of healthcare workers are at equal risk of carriage. We aimed to explore S. aureus nasal carriage among healthcare professionals.

Methods. Prospective study conducted in two French university hospitals in 2014 and 2016. Volunteers were screened for S. aureus nasal carriage. Profession and hygiene habits were collected. Based on the results of this initial study, a second study focused on semi-skilled workers and biomedical equipment technicians (BETs) only; participants were given education on the basic rules of hygiene, then re-screened three months later.

Results. In the initial study, $38.8 \%$ of the 436 participants were detected as nasal carriers. There was a significant difference in nasal carriage according to professional category $(p<0.0001)$; the lowest was found among administrative agents (17.3\%), followed by healthcare providers $(37.4 \%)$, laboratory technicians $(37.6 \%)$. The greatest proportion was found among semi-skilled workers and BETs (52.9\%). Spa-typing ruled out the hypothesis of a single clone dissemination among colleagues. After the three-month hygiene awareness campaign, all re-screened individuals remained positive, and with their respective initial strain.

Conclusions. To the best of our knowledge we report here for the first time that semi-skilled workers and BETs are specifically more at risk of $S$. aureus nasal colonisation. This striking finding urges hospital hygiene departments to evaluate this specific professional category and implement strategies to improve hygiene awareness.

Keywords: Staphylococcus aureus; nasal carriage; healthcare workers 


\section{INTRODUCTION}

Staphylococcus aureus (S. aureus) is known as a major human pathogen causing a wide range of nosocomial and community-acquired infections. Other than its pathogenicity, S. aureus is also a human commensal that is retrieved from many different body sites such as the nose, throat, digestive tractus, perineum, and skin surface (Acton et al., 2009; Bourgeois-Nicolaos et al., 2010; Claassen-Weitz et al., 2016; Wertheim et al., 2005). Notably, the nares represent the main reservoir of S. aureus carriage; around $30 \%$ of the general population being colonised (Kluytmans et al., 1997). It has been shown that some individuals host S. aureus on a regular basis (known as persistent carriers), while other carriers have only a transient colonisation (intermittent carriers) (Wertheim et al., 2005). It is well known that $S$. aureus nasal carriage is associated with an increased risk of developing subsequent $S$. aureus infections (Eiff et al., 2001; Wertheim et al., 2005), in particular for persistent carriers (Verhoeven et al., 2014), in both community-acquired and healthcare associated infections (Kluytmans et al., 1997; Muñoz et al., 2008).

Nasal colonisation rate relies on both host (immune response, nasal microbiota, antimicrobial peptides release) and bacterial (adhesins, ability to survive in stress or deprived conditions) determinants (Johannessen et al., 2012; Peacock et al., 2001). Some risk factors for nasal colonisation have been extensively described and include promiscuous living conditions (military conditions, fire station, etc...), older age, male sex, or patients undergoing haemodialysis (Cole et al., 2001; Heijer et al., 2013; Herwaldt et al., 2004; Yu et al., 1986). While repeated exposure to $S$. aureus due to frequent contact with patients or microbiological substances may increase carriage risk (Okamo et al., 2016; van Vugt et al., 2015), it has been described that the compliance with basic hygiene recommendations, including alcohol-based hand rub solution (AHS), reduces the risk of nasal colonisation (Gould et al., 2018; Saadatian- 
Elahi et al., 2013). However, few studies have explored S. aureus nasal colonisation and inherent risk factors among the various categories of hospital staff. A recent paper has reported that medical technologists (i.e. those working in operating rooms, chemotherapy, and imaging) had a higher nasal carriage rate than other healthcare workers (HCW) in the same hospital in Argentina (Boncompain et al., 2017). Also, van Vugt et al. found that S. aureus nasal carriage rate was significantly greater among surgeons compared to non-hospitalised individuals (van Vugt et al., 2015). These elements suggest that the hospital setting may be a favouring condition for S. aureus nasal carriage, but with disparities between HCW categories, and S. aureus transmission by colonised HCW. The aim of the present study was to explore S. aureus nasal carriage among different HCW categories in two independent tertiary hospitals in France.

\section{METHODS}

Written informed consent was obtained from all participants. The studies were conducted in accordance with the declaration of Helsinki, French national regulations, and approved by the local ethics committee that considered the study as non-interventional. Relevant approval (reference RPD09891439) regarding access to patient-identifiable information was granted by the French data protection agency (Commission Nationale Informatique et Liberté, CNIL).

Information posters advertised the study throughout two French university hospitals (Lyon and Grenoble, in April 2014 and June 2016, respectively), inviting all individuals among the personnel to participate. Those who volunteered underwent nasal swabbing on one of two consecutive days. Double plastic swabs were used; each nostril was sampled by both swabs (Copan, Brescia, Italy). S. aureus carriage was screened by real-time PCR $\left(\right.$ GenXpert ${ }^{\circledR}$ SA Nasal Complete, Cepheid, Maurens-Scopont, France) with the first swab. For positive 
samples, the second swab was subsequently cultured on ChromAgar ${ }^{\circledR}$ (I2A, Montpellier, France) and blood agar plate (bioMérieux, Marcy-l'étoile, France; $35^{\circ} \mathrm{C}, 48 \mathrm{~h}$ ). Identification of suspect colonies was confirmed by either both catalase and latex agglutination tests (Slidex, bioMérieux, Marcy-l'étoile, France) or MALDI-TOF. S. aureus strains were stored at $-20^{\circ} \mathrm{C}$. An anonymous questionnaire collecting demographic data, profession, risk factors like haemodialysis, immunosuppressive treatment, diabetes mellitus, and type of contact with patients was filled out by each participant. Profession categories were grouped as follows: (i) HCWs without direct contact with patients and without exposure to the hospital or laboratory environment (i.e. director of human resources, clinical research associate, lawyer, etc...) hereafter termed "administrative agents", (ii) HCWs with direct contact with patients and exposed to the hospital environment (i.e. nurses, physicians, physical therapists, dieticians, radio technicians, psychologists, stretcher bearers, etc...) hereafter termed "healthcare providers", (iii) HCWs without direct contact with patients and exposed to the laboratory environment hereafter termed "lab technicians" and (iv) HCWs without direct contact with patients and exposed to the hospital environment (i.e. semi-skilled workers such as carpenters, electricians, plumbers, and biomedical equipment technicians (BETs) in charge of the repair and maintenance of medical instruments) hereafter termed "semi-skilled workers and BETs".

A second study focusing on semi-skilled workers and BETs only, was conducted in 2015 in Lyon, for which volunteers underwent nasal swabbing based on the same design as the initial study. To maximise the sensitivity of the screening, cultures were systematically performed along with molecular testing. A worker was considered as carrier when at least one of the two techniques was positive. The anonymous questionnaire was enriched with an item on hand hygiene habits. Following the initial nasal swabbing, participants were given weekly educational sessions on basic recommendations of hand hygiene by the hospital hygiene team and the occupational health department and were supplied with individual bottles of AHS 
(replaced on demand) for a 3-month period. A final nasal screening was performed after three months. S. aureus strains isolated from positive samples were characterised by spa-typing according to the Ridom Staph Type standard protocol (http://www.ridom.com).

Categorical variables were reported as number and percentage, and continuous variables are reported as median and interquartile range [IQR]. Participant characteristics were compared using Chi-squared or Fisher's exact tests for categorical variables. Quantitative variables were compared using Mann-Whitney tests. All statistical tests were two-tailed and a P value $\leq 0.05$ was considered statistically significant. Statistics was performed with Stata, version 12 (Stata Corp, Texas, USA). Because gender influences $S$. aureus carriage rate and the sex ratios differed between professional groups, we determined whether between-group differences could result from sex ratio differences. The associations between $S$. aureus carriage and professional group were analyzed in a mixed-effect logistic model including patient sex and the hospital (Grenoble or Lyon) as random effects. Computations were performed using packages lme4 and lmerTest for the R environment version 3.6.0.

\section{RESULTS}

A total of 309 and 157 HCWs participated in the study in Lyon (2014) and Grenoble (2016). Respectively, 7 and 16 participants in Lyon and Grenoble were excluded due to invalid molecular tests; a further 7 participants in Lyon were excluded due to missing questionnaires. A total of 436 individuals were thus included in the study, 295 individuals in Lyon and 141 in Grenoble. Two-thirds $(67.7 \%)$ of the total population (68.4\% in Lyon, $66.4 \%$ in Grenoble) were women. The median age was 43 years (IQR [31-53]) and this was significantly higher for participants in Lyon (45 years, IQR [33-54]) than Grenoble (39 years, IQR [27-50]; p = 
0.0012)(Table 1). Participants were distributed as follows: $41 / 578$ and 11/482 administrative agents, 94/4100 and 53/4155 healthcare providers, 109/140 and 24/277 lab technicians, and 51/380 and 53/242 semi-skilled workers and BETs in Lyon and Grenoble, respectively.

Overall, 38.8\% ( $\mathrm{n}=169)$ of the participants were detected as $S$. aureus nasal carriers, with a significantly higher proportion in Grenoble $(46.8 \%, \mathrm{n}=66)$ than in Lyon $(34.9 \%, \mathrm{n}=103$; $\mathrm{p}=0.02$, Table 1). Only 2 strains were detected as methicillin-resistant $S$. aureus (MRSA) in Lyon $(0.7 \%)$, and none in Grenoble.

There was a significant difference in the proportion of nasal carriage according to professional category $(\mathrm{p}<0.0001)$; the lowest proportion was found among administrative agents $(17.3 \%)$, followed by healthcare providers (37.4\%), and laboratory technicians (37.6\%). Unexpectedly, the greatest proportion was found among semi-skilled workers and BETs (52.9\%; Table 1). These results were confirmed when controlling for sex and sampling location. Indeed, compared to the healthcare providers, the frequency of $S$. aureus carriage was similar in laboratory technicians (adjusted $\mathrm{OR}=0.95,95 \% \mathrm{CI} 0.56$ to $1.61 \mathrm{p}=0.86$ ), lower in administrative agents (adjusted $\mathrm{OR}=0.35,95 \%$ CI 0.15 to $0.91, \mathrm{p}=0.015$ ), higher in semiskilled workers and BETs (adjusted $\mathrm{OR}=1.77,95 \%$ CI 1.02 to 3.06, $\mathrm{p}=0.042$ ).

To explore further this observation, the second study focused on this specific HCWs category in Lyon in 2015. Among the 44 semi-skilled workers and BETs who participated, 23 (52.4\%) were $S$. aureus nasal carriers (none MRSA) at the initial swabbing (M0), confirming the results of the initial study (Figure 1); 20 were detected by both PCR and culture, and 3 by culture only ( 2 invalid and 1 discordant negative molecular tests).

At M0, the 23 isolates belonged to 19 different spa-types (Figure 2). No predominant spatype was observed; a maximum of two individuals harbouring the same spa-type ( $\mathrm{t} 2$, $\mathrm{t} 148$, t342, and t1451)(Table 2). After 3 months, 22 of the 44 participants accepted to be re- 
screened; 14 of which were initially known as carriers and 8 as non-carriers. All the 14 carriers were still positive for nasal $S$. aureus after 3 months, 6 of the 8 non-carriers remained negative, and 2 acquired $S$. aureus over the 3-month period (Figure 1). Characterisation of the 16 isolated strains by spa-typing found 12 different spa-types ( 2 failures); the 14 participants who were still detected positive harboured their respective initial strain, based on spa-type (Table 2).

All the 44 semi-skilled workers and BETs enrolled in this second study declared having no direct contact with patients or microorganisms. All of them also affirmed "never" or "rarely" wearing gloves and using AHS when on duty. After 3 months, 90.9\% $(n=20)$ of the participants who were re-screened declared now using AHS "often" or "always" after each intervention.

\section{DISCUSSION}

Although $S$. aureus nasal carriage is extensively explored, few studies have investigated $S$. aureus nasal colonisation and inherent risk factors among HCWs. The present study found an overall carriage rate of $38.8 \%$ in two French university-hospital personnel, which is above usually stated rates in the general population, which is around 30\% (Kluytmans et al., 1997; Lepelletier and Lucet, 2013).

Strikingly, when stratifying by professional categories, we found that semi-skilled workers and BETs had a significantly higher carriage rate, with about half of the group being colonised. To the best of our knowledge, this is the very first time that such a professional category has been investigated. Interestingly, similar results were obtained in the second study, and in both independent hospitals, strengthening the validity of this finding. Data were controlled for sex and hospital site, ruling out such possible biases. Other risk factors like 
haemodialyssis, immunosuppressive treatment or diabetes mellitus were exceptional among participants, ruling out a confounding factor.

Moreover, the hypothesis of a single clone dissemination among professionals was ruled out since a wide variety of spa-types were observed in this group. One may hypothesise that such hospital professionals, while exposed to a microbiologically contaminated environment on a daily basis, do not comply with hygiene recommendations as strictly as medical and paramedical staff. Strikingly, all the semi-skilled workers and BETs declared in the second study no direct contact with patients or microbiological agents, while in fact working within the patient care environment when intervening in patient rooms or medical wards for technical reasons. Moreover, at the beginning of the second study, all declared "never" or "rarely" wearing gloves or using AHS when on duty. This highlights the under-recognition of the microbiological risk associated with their conditions of work. The combination of both exposure to a contaminated environment and lack of knowledge and compliance with hand hygiene rules may be determinants for such a high carriage rate among these professionals. This is supported by the finding that healthcare providers, who are in direct contact with patients but made aware of importance of hand hygiene, displayed a lower carriage rate. In addition, administrative agents who work in a separate dedicated building, and thus have no direct contact with patients and no exposure to the hospital environment, showed the lowest carriage rate.

The impact of the 3-month educational course on hygiene was evaluated by both the selfreported hygiene habits and their rate of nasal carriage. Almost all of the participants declared increased hygiene compliance over the 3-month period, with a virtually systematic use of AHS after a technical intervention. Nevertheless, there was no decrease of carriage rate over the study period since all the carriers who underwent nasal swabbing at both inclusion time and 3 months later remained positive. Since persistent carriers represent only $20-25 \%$ of the 
carrier population (Verhoeven et al., 2012), the lack of impact of the 3-month educational course on hygiene could not be attributed to such a hypothesis. However, as the change in hygiene habits over the study period was only based on participant declaration, the accuracy of such declarations and the effective progress of compliance to basic hygiene recommendations could be questioned. More generally, the results of the present study lead to the question of the risk of $S$. aureus transmission in the hospital setting posed by such colonised workers, but also the determinants of $S$. aureus nasal carriage, which could include socio-economic status and the level of education that may be of interest to investigate in future studies.

Regarding the limitations of the study, it would be interesting to perform similar studies at a greater-scale. Also, we are aware that some people would more gladly participate than others, which could induce a bias, even though we considered that such volunteers would not be associated with a significantly different carriage rate.

To the best of our knowledge we report here for the first time that semi-skilled workers and BETs are specifically more at risk of $S$. aureus nasal colonisation. This striking finding urges hospital hygiene departments to evaluate this specific professional category and implement strategies to improve hand hygiene awareness.

\section{DECLARATION OF INTEREST}

None

\section{FUNDING}

This work was supported by no external funding. However, PCR tests (GenXpert ${ }^{\circledR}$ SA Nasal Complete) for the first study in 2014 in Lyon were supplied by Cepheid France. Cepheid had neither a role in the design of the study nor in the analysis of the data and the writing of the manuscript. 


\section{ACKNOWLEDGMENTS}

The authors are sincerely grateful to the technicians of the French National Reference Centre for Staphylococci and the bacteriology clinical laboratory teams in Lyon and Grenoble for their technical support. We also acknowledge the managers of the biomedical equipment technicians department for their cooperation. Finally, we are thankful to Philip Robinson for his precious guidance in the writing of the manuscript and his expertise in English spelling. Parts of the results of the study were presented in a poster at the Réunion Interdisciplinaire de Chimiothérapie Anti-Infectieuse 2018 in Paris.

\section{FIGURE CAPTIONS}

Figure 1. Flowchart of semi-skilled workers and biomedical equipment technicians participating in the second study (Lyon, 2015). Volunteers among semi-skilled workers and BETs underwent nasal swabbing and were then given weekly educational sessions on basic recommendations of hand hygiene for a 3-month period. A final nasal screening was performed after three months.

Figure 2. Distribution of the $23 \mathrm{~S}$. aureus strains isolated from of semi-skilled workers and biomedical equipment technicians participating in the second study (Lyon, 2015) based on spa-types. Each circle represents a carrier participant. Among the 23 S. aureus carriers, 19 different spa-types were detected, 10 of which were singletons. 


\section{REFERENCES}

Acton, D.S., Plat-Sinnige, M.J.T., van Wamel, W., de Groot, N., van Belkum, A., 2009. Intestinal carriage of Staphylococcus aureus: how does its frequency compare with that of nasal carriage and what is its clinical impact? Eur. J. Clin. Microbiol. Infect. Dis. 28, 115-127. doi:10.1007/s10096-008-0602-7

Boncompain, C.A., Suárez, C.A., Morbidoni, H.R., 2017. Staphylococcus aureus nasal carriage in health care workers: First report from a major public hospital in Argentina. Rev. Argent. Microbiol. 49, 125-131. doi:10.1016/j.ram.2016.12.007

Bourgeois-Nicolaos, N., Lucet, J.-C., Daubié, C., Benchaba, F., Rajguru, M., Ruimy, R., Andremont, A., Armand-Lefevre, L., 2010. Maternal vaginal colonisation by Staphylococcus aureus and newborn acquisition at delivery. Paediatr Perinat Epidemiol 24, 488-491. doi:10.1111/j.1365-3016.2010.01139.x

Claassen-Weitz, S., Shittu, A.O., Ngwarai, M.R., Thabane, L., Nicol, M.P., Kaba, M., 2016. Fecal Carriage of Staphylococcus aureus in the Hospital and Community Setting: A Systematic Review. Front Microbiol 7, 449. doi:10.3389/fmicb.2016.00449

Cole, A.M., Tahk, S., Oren, A., Yoshioka, D., Kim, Y.H., Park, A., Ganz, T., 2001. Determinants of Staphylococcus aureus nasal carriage. Clin. Diagn. Lab. Immunol. 8, 1064-1069. doi:10.1128/CDLI.8.6.1064-1069.2001

Eiff, von, C., Becker, K., Machka, K., Stammer, H., Peters, G., 2001. Nasal carriage as a source of Staphylococcus aureus bacteremia. Study Group. N Engl J Med 344, 11-16. doi:10.1056/NEJM200101043440102

Gould, D., Moralejo, D., Drey, N., Chudleigh, J., Taljaard, M., 2018. Interventions to improve hand hygiene compliance in patient care: Reflections on three systematic reviews for the Cochrane Collaboration 2007-2017. J Infect Prev 19, 108-113. doi:10.1177/1757177417751285 
Heijer, den, C.D.J., van Bijnen, E.M.E., Paget, W.J., Pringle, M., Goossens, H., Bruggeman, C.A., Schellevis, F.G., Stobberingh, E.E., APRES Study Team, 2013. Prevalence and resistance of commensal Staphylococcus aureus, including meticillin-resistant S aureus, in nine European countries: a cross-sectional study. Lancet Infect Dis 13, 409-415. doi:10.1016/S1473-3099(13)70036-7

Herwaldt, L.A., Cullen, J.J., French, P., Hu, J., Pfaller, M.A., Wenzel, R.P., Perl, T.M., 2004. Preoperative risk factors for nasal carriage of Staphylococcus aureus. Infect Control Hosp Epidemiol 25, 481-484. doi:10.1086/502426

Johannessen, M., Sollid, J.E., Hanssen, A.-M., 2012. Host- and microbe determinants that may influence the success of S. aureus colonization. Front Cell Infect Microbiol 2, 56. doi:10.3389/fcimb.2012.00056

Kluytmans, J., van Belkum, A., Verbrugh, H., 1997. Nasal carriage of Staphylococcus aureus: epidemiology, underlying mechanisms, and associated risks. Clin. Microbiol. Rev. 10, $505-520$.

Lepelletier, D., Lucet, J.-C., 2013. Controlling meticillin-susceptible Staphylococcus aureus: not simply meticillin-resistant S. aureus revisited. J. Hosp. Infect. 84, 13-21. doi:10.1016/j.jhin.2013.01.010

Muñoz, P., Hortal, J., Giannella, M., Barrio, J.M., Rodríguez-Créixems, M., Pérez, M.J., Rincón, C., Bouza, E., 2008. Nasal carriage of S. aureus increases the risk of surgical site infection after major heart surgery. J. Hosp. Infect. 68, 25-31. doi:10.1016/j.jhin.2007.08.010

Okamo, B., Moremi, N., Seni, J., Mirambo, M.M., Kidenya, B.R., Mshana, S.E., 2016. Prevalence and antimicrobial susceptibility profiles of Staphylococcus aureus nasal carriage among pre-clinical and clinical medical students in a Tanzanian University. BMC Res Notes 9, 47. doi:10.1186/s13104-016-1858-0 
Peacock, S.J., de Silva, I., Lowy, F.D., 2001. What determines nasal carriage of Staphylococcus aureus? Trends Microbiol 9, 605-610.

Saadatian-Elahi, M., Tristan, A., Laurent, F., Rasigade, J.-P., Bouchiat, C., Ranc, A.-G., Lina, G., Dauwalder, O., Etienne, J., Bes, M., Vandenesch, F., 2013. Basic rules of hygiene protect health care and lab workers from nasal colonization by Staphylococcus aureus: an international cross-sectional study. PLoS ONE $\quad 8, \quad$ e82851. doi:10.1371/journal.pone.0082851

van Vugt, J.L.A., Coelen, R.J.S., van Dam, D.W., Winkens, B., Derikx, J.P.M., Heddema, E.R., Stoot, J.H.M.B., 2015. Nasal carriage of Staphylococcus aureus among surgeons and surgical residents: a nationwide prevalence study. Surg Infect (Larchmt) 16, 178182. doi:10.1089/sur.2014.022

Verhoeven, P.O., Gagnaire, J., Botelho-Nevers, E., Grattard, F., Carricajo, A., Lucht, F., Pozzetto, B., Berthelot, P., 2014. Detection and clinical relevance of Staphylococcus aureus nasal carriage: an update. Expert Rev Anti Infect Ther 12, 75-89. doi:10.1586/14787210.2014.859985

Verhoeven, P.O., Grattard, F., Carricajo, A., Lucht, F., Cazorla, C., Garraud, O., Pozzetto, B., Berthelot, P., 2012. An algorithm based on one or two nasal samples is accurate to identify persistent nasal carriers of Staphylococcus aureus. Clin. Microbiol. Infect. 18, 551-557. doi:10.1111/j.1469-0691.2011.03611.x

Wertheim, H.F.L., Melles, D.C., Vos, M.C., van Leeuwen, W., van Belkum, A., Verbrugh, H.A., Nouwen, J.L., 2005. The role of nasal carriage in Staphylococcus aureus infections. Lancet Infect Dis 5, 751-762. doi:10.1016/S1473-3099(05)70295-4

Yu, V.L., Goetz, A., Wagener, M., Smith, P.B., Rihs, J.D., Hanchett, J., Zuravleff, J.J., 1986. Staphylococcus aureus nasal carriage and infection in patients on hemodialysis. Efficacy of antibiotic prophylaxis. N Engl J Med 315, 91-96. 
doi:10.1056/NEJM198607103150204 


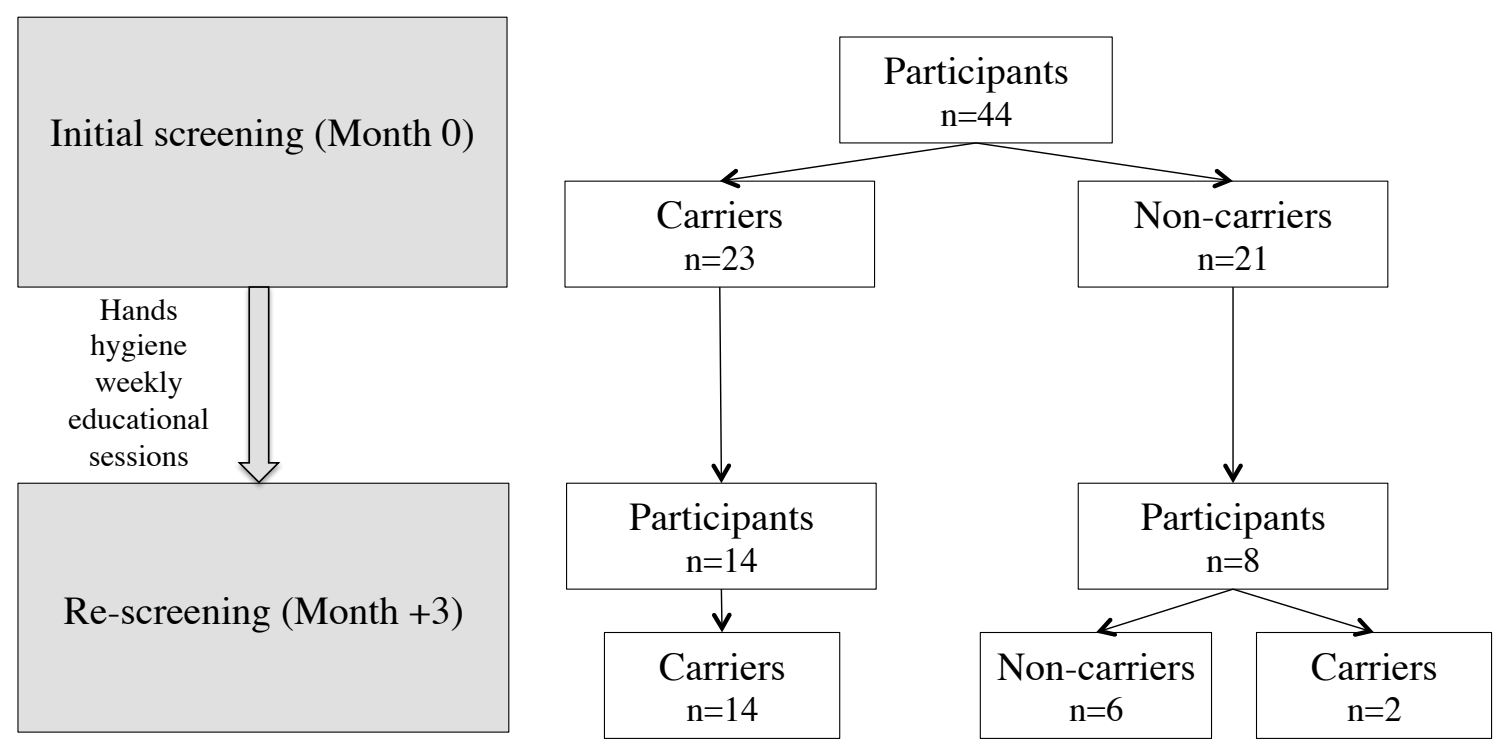




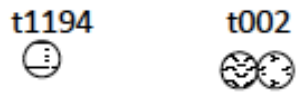

$\Theta^{\mathrm{t} 018}$

t084

풍

t189

(2)

$$
\Leftrightarrow \mathrm{t}^{\mathrm{B} 239} \mathrm{~g}
$$

t280

丹

t7880

DEs 8

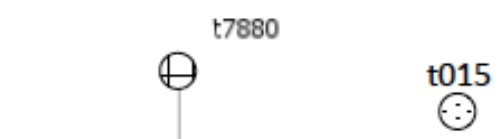

t1187

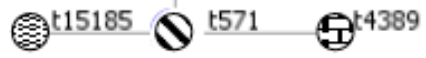

$O^{t 645} \bigcirc^{t 272}$

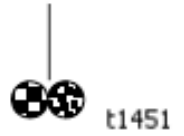

t148

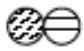




\begin{tabular}{|c|c|c|c|c|}
\hline & \multirow{2}{*}{$\begin{array}{c}\text { Total } \\
\text { population } \\
\text { n=436 }\end{array}$} & \multicolumn{2}{|c|}{ Hospital } & \multirow[b]{2}{*}{$P$ value } \\
\hline & & $\begin{array}{l}\text { Lyon } \\
n=295\end{array}$ & $\begin{array}{c}\text { Grenoble } \\
\text { n=141 }\end{array}$ & \\
\hline Female sex, n (\%) & $275(67.7)$ & $186(68.4)^{(\mathrm{a})}$ & $89(66.4)^{(b)}$ & NS \\
\hline Median age, years [IQR] & $43[31-53]$ & $45[33-54]^{(\mathrm{c})}$ & $39[27-50]^{(\mathrm{d})}$ & 0.0012 \\
\hline \multicolumn{5}{|l|}{ Nasal carriers, $n(\%)$} \\
\hline Administrative agents ${ }^{(\mathrm{e})}$ & $9(17.3)^{* * *}$ & $7(17.1)$ & $2(18.2)$ & NS \\
\hline Healthcare providers ${ }^{(\mathrm{f})}$ & $55(37.4)^{* * * *}$ & $34(36.2)$ & $21(39.6)$ & NS \\
\hline Lab technicians & $50(37.6)^{* * * *}$ & $39(35.8)$ & $11(45.8)$ & NS \\
\hline Semi-skilled workers ${ }^{(\mathrm{g})}$ and & $55(52.9)^{* * *}$ & $23(45.1)$ & $32(60.4)$ & NS \\
\hline \multicolumn{5}{|l|}{ BETs } \\
\hline Total & $169(38.8)$ & $103(34.9)$ & $66(46.8)$ & 0.02 \\
\hline
\end{tabular}

Abbreviations: IQR : interquartile range; NS : non significant; BETs : biomedical equipment technicians.

(a) 23 missing data

(b) 6 missing data

(c) 26 missing data

(d) 6 missing data

(e) director of human resources, clinical research associate, jurist, etc...

(f) nurses, physicians, physical therapists, dieticians, radio technicians, psychologists, stretcher bearers, etc...

(g) carpenters, electricians, plumbers and biomedical equipment technicians

*** $\mathrm{p}<0.0001$. The rate of nasal carriage in the semi-skilled workers and BETs group was significantly higher than in each other group. 
Table 2. Characterisation by spa-typing of strains carried by semi-skilled workers and biomedical equipment technicians in the second study (Lyon, 2015)

\begin{tabular}{ccc}
\hline $\begin{array}{c}\text { Participant ID } \\
\text { number }\end{array}$ & Spa-type at Month 0 & Spa-type at Month +3 \\
\hline 1 and 6 & 148 & NRS \\
2 & 280 & NRS \\
3 & 272 & NRS \\
4 & 15185 & 15185 \\
5 & 15 & 15 \\
7 & 21 & NRS \\
8 & 571 & 571 \\
9 & 189 & NRS \\
10 & 18 & failure \\
11 & 645 & NRS \\
12 & 15187 & 15187 \\
13 and 22 & 1451 & 1451 \\
14 & 342 & 342 \\
15 & 1239 & 1239 \\
16 and 21 & 2 & 2 and NRS \\
17 & 7880 & 7880 \\
18 & 1194 & 1194 \\
19 & 342 & 342 \\
20 & 4389 & NRS \\
23 & 84 & 84 \\
24 & N/A & 148 \\
25 & N/A & failure \\
\hline
\end{tabular}

Abbreviations: N/A: Not Applicable (non-carrier participant); NRS: participant not rescreened

Failure: no spa-type assignable 